\title{
Association between sleep-disordered breathing, sleep-wake pattern, and cognitive impairment among patients with chronic heart failure
}

\author{
Carina Hjelm, Anna Strömberg, Kristofer Franzén Årestedt and Anders Broström
}

\section{Linköping University Post Print}

\section{Tweet}

N.B.: When citing this work, cite the original article.

Original Publication:

Carina Hjelm, Anna Strömberg, Kristofer Franzén Årestedt and Anders Broström, Association between sleep-disordered breathing, sleep-wake pattern, and cognitive impairment among patients with chronic heart failure, 2013, European Journal of Heart Failure, (15), 5, 496-504.

http://dx.doi.org/10.1093/eurjhf/hft014

Copyright: The Authors http://www.oxfordjournals.org/

Postprint available at: Linköping University Electronic Press http://urn.kb.se/resolve?urn=urn:nbn:se:liu:diva-93967 


\section{Association between sleep disordered breathing, sleep- wake pattern and cognitive impairment among patients with chronic heart failure}

Carina Hjelm ${ }^{1,2}$, Anna Strömberg ${ }^{1,3}$, Kristofer Årestedt ${ }^{1,4}$, Anders Broström ${ }^{5,6}$

Department of Medical and Health Sciences, Division of Nursing Science, Linköping University, S-58185 Linköping, Sweden ${ }^{1}$

Department of Cardiothoracic Surgery, Linköping University Hospital ${ }^{2}$

Department of Cardiology, Linköping University Hospital, Sweden ${ }^{3}$

School of Health and Caring Sciences, Linnaeus University Kalmar, Sweden ${ }^{4}$

Department of Clinical Neurophysiology, Linköping University Hospital ${ }^{5}$

Department of Nursing Science, School of Health Sciences, Jönköping University ${ }^{6}$

Corresponding author: Carina Hjelm, tel.+46-10-1031787, fax. +46-13123285, e-mail carina.hjelm@liu.se 
Background: Chronic Heart failure (CHF) and sleep disordered breathing (SDB) are often coexisting problems among the elderly. Apnoeic events may cause cognitive impairment. The aim of the study was to compare sleep and wake patterns, insomnia, daytime sleepiness and cognitive function in community- dwelling CHF patients, with and without SDB, and to investigate the association between sleep-related factors and cognitive dysfunction.

Methods: In this cross-sectional observational study, SDB was measured with Apnea Link and defined as an Apnea-Hypopnea Index (AHI) $\geq 15 /$ hour of sleep. Sleep and wake patterns were measured with actigraphy for one week. Insomnia was measured with Minimal Insomnia Symptom Scale, daytime sleepiness with Epworth Sleepiness Scale and cognitive function with a neuropsychological test battery.

Results: 137 patients (68\% male, median age 72 years, 58\% NYHA II) were consecutively included. Forty-four percent had SDB (AHI $\geq 15)$. The SDB group had significantly higher saturation time below $<90 \%$, more difficulties maintaining sleep and lower levels of daytime sleepiness compared to the non- SDB group. Cognitive function and sleep and wake patterns did not differ between the SDB and the non- SDB group. Insomnia was associated with decreased global cognition.

Conclusion: The prevalence of cognitive dysfunction was low in this population with predominantly mild to moderate CHF. This might have influenced the lack of associations between cognitive function and SDB. Insomnia was the only sleep-related factor significantly influencing cognition.

Keywords: chronic heart failure, cognitive dysfunction, sleep disordered breathing, sleepwake pattern, insomnia 


\section{Introduction}

Sleep disordered breathing (SDB) occurs in more than seven out of ten chronic heart failure (CHF) patients in New York Heart Association (NYHA) class $\geq$ II, when using an apnoea hypopnoea index $(\mathrm{AHI}) \geq 5 / \mathrm{h} .{ }^{1}$ Patients with $\mathrm{CHF}$ and SDB often report disturbed sleep and wake patterns with shorter total sleep time, markedly reduced sleep efficiency, as well as longer sleep onset latency and longer wake time after sleep onset compared to patients without SDB. ${ }^{2}$ Other consequences include insomnia (i.e., difficulties initiating sleep, difficulties maintaining sleep and non- restorative sleep) and excessive daytime sleepiness. ${ }^{3,4}$ Sleep loss and sleep fragmentation are in other contexts known to affect cognitive functions such as memory encoding, consolidation, plasticity and reconsolidation. ${ }^{5}$ Furthermore, executive functions, including decision-making processes ${ }^{6}$, as well as attention and psychomotor speed are also affected. ${ }^{7,8}$

It has been described that elderly individuals with mild to moderate CHF often have a mild cognitive dysfunction. ${ }^{9}$ Recent studies ${ }^{8,10,11}$ in adults (mean age $62.8 \mathrm{yrs}$ ) with cardiovascular disease show that “poor sleep” (i.e., global score $\geq 5$ on the Pittsburgh Sleep Quality Index) is independently associated with reduced global cognitive function ${ }^{10}$ and executive functioning and attention ${ }^{11}$. Other studies ${ }^{12,13,14}$ not focusing on CHF show that SDB-related hypoxia can be an indicator associated with cognitive dysfunction and dementia. Furthermore, reduced quality of life, anxiety and depression have also been described. ${ }^{15,11}$ Sleep disturbances, such as SDB, may from a pathophysiological perspective affect the development of early atherosclerosis (i.e., by an increased sympathetic activation), and as a consequence increase the risk of vascular dementia in patients with $\mathrm{CHF} .{ }^{16}$ However, few if any studies have 
investigated the effects of SDB, insomnia and sleep-wake patterns on cognitive function in patients with CHF. The aim of the study was (I) to describe sleep and wake patterns, insomnia, excessive daytime sleepiness and cognitive function in community dwelling CHF patients with and without SDB and (II) to investigate the association between sleep-related factors and cognitive dysfunction.

\section{Methods}

\subsection{Design and setting}

This study has a cross-sectional design. A total of 137 stable consecutive patients with CHF living in the community were recruited after discharge from outpatient clinics at one university hospital and two county hospitals in the south of Sweden. Inclusion criteria were being 18 years of age and suffering from CHF, based on the European Society of Cardiology

guidelines. ${ }^{17}$ Exclusion criteria were severe psychiatric illness, a history of neurological disorders (e.g., Alzheimer's disease, vascular or mixed dementia and severe head injury), drug abuse or difficulties reading and understanding the Swedish language. No patients within nursing homes or advanced home care were included.

\subsection{Procedures}

Demographic and clinical variables such as age, gender, marital status, cardiac function, medications, laboratory data and co-morbidities were collected by a study nurse during a clinical examination and from medical records. Laboratory data included erythrocytes, erythrocyte volume fraction, hemoglobin, leucocytes, thrombocytes, sodium, potassium, creatinine, albumin, transferrin, transthyretin, and B-type natriuretic peptide (BNP). CHF was classified with NYHA class II-IV. Diabetes diagnoses were based on a history of diabetes, 
and/or current treatment (oral therapy or insulin). Hypertension diagnoses were based on a blood pressure level of more than 140/90 mmHg. During the week that followed, patients answered questionnaires and performed registrations of SDB and sleep-wake pattern. After one week, a specialist educated/trained nurse performed the cognitive tests in the patients' homes or at the hospital. All data were collected between September 2009 and December 2011. The study was approved by the Ethics Committee (study code M222-08/T81-09) and conducted in accordance with the Declaration of Helsinki.

\section{Measurements}

\section{Sleep and breathing}

\section{3:1. Sleep disordered breathing}

Full night respiratory recordings were performed in the patients' homes using a portable twochannel screening tool (ApneaLink device, ResMed Corporation, Poway, CA, USA). ApneaLink measures airflow through a nasal cannula connected to a pressure transducer and pulse oximetry. Apnoeas and hypopneas are automatically scored by the ApneaLink software and an AHI is provided based on recording time. Validation of the ApneaLink demonstrates that the device provides reliable information regarding AHI, when compared with a full polysomnography. ${ }^{18} 19$ SDB was dichotomized into two groups; no SDB (AHI < 15/hour of sleep) and SDB (AHI $\geq 15 /$ hour of sleep).

\subsection{Sleep and wake pattern}

A one- week registration of the patients' sleep and wake pattern was performed using actigraphy (SenseWear BMS Pro 2, BodyMedia Inc, Pittsburg, PA, USA). The SenseWear BMS is a simple, wireless device that is worn on the upper right arm under the clothing for 
continuous data collection. It is a valid and reliable tool that reveals more fragmented sleep than polysomnography. ${ }^{20}$

\subsection{Excessive daytime sleepiness and insomnia}

3:3.1 Epworth Sleepiness Scale (ESS) consists of 8 items that describe different daily situations where the patients are asked to rate the likeliness of dozing off or falling asleep. Each situation is rated on a scale of $0-3$, where high scores indicate a greater propensity to fall asleep. The total score is 24 , with a cut off $>10$ indicating excessive daytime sleepiness. ${ }^{21}$ The instrument has shown good validity and reliability across different age groups and populations. $^{22}$

3.3.2 Minimal Insomnia Symptom Scale (MISS) consists of three items focusing on difficulties initiating sleep, difficulties maintaining sleep and non-restorative sleep. ${ }^{23,24}$ The patients grade their difficulties on a scale ranging from no problems (0), to very great problems (4). Using a summation score, insomnia can be sub-grouped as no insomnia (0-3), subclinical insomnia (4-6), moderate insomnia (7-9) and severe insomnia (10-12). Results have shown that MISS possesses adequate reliability and validity among the elderly. ${ }^{25}$

\subsection{Cognitive function}

A battery of six well validated tests was used to measure different aspects of cognitive function.

3.4.1 Global cognitive function was measured with Mini-Mental State Examination (MMSE). This is a cognitive screening test with a maximum score of 30 . The instrument contains 
questions about different abilities such as orientation, attention and concentration, sequence, visual-spatial abilities, verbal learning and memory, reading aloud, understanding and verbal repetition. A score $\geq 28$ is considered to indicate normal cognitive function, $25-27$ mild cognitive dysfunction whereas MMSE $\leq 24$ is considered as cognitive dysfunction with a high risk for dementia. ${ }^{26}$

3.4.2 Psychomotor speed was measured by Trail making $A .{ }^{27}$ The test reports the number of seconds required to complete the task, higher scores reveal greater impairment. The Trail making $B$ was used to test executive function, but in alternate alpha-numeric order (i.e. numberletter-number-letter). The measure of analysis is the number of seconds required to complete the task. Longer time indicates poorer shifting between numbers and letters. ${ }^{28}$

3.4.3 Visual-spatial perception/construct and memory was measured by Rey Ostereich Complex Figure (ROCF). Examinees are asked to reproduce a complicated line drawing, first by copying and then from memory (immediate recall and delayed recall), with a maximum score of 36. Examinees are not told beforehand that they will be asked to draw the figure from memory, since the immediate and delayed recall conditions are a test of incidental or implicit memory. ${ }^{29}$

3.4.4 Semantic memory was measured with the Word knowledge test. The examinee receives a list with a total of 30 words. With each listed words, he/she is instructed to make a choice between five examples, one of which has the same meaning as the word to the left. The maximum score is 30 if all the words are correct and performed within 7 minutes. ${ }^{30}$

3.4.5 The Memory of a story test was used for measuring episodic memory. A predetermined short story is read and the number of points is based on immediate (strict and liberal) or delayed (strict and liberal) response with a total of 21 points for each section. After 20 minutes 
the same test is performed again to test the recall of the same story. ${ }^{31}$

3.4.6 Spatial performance was measured with the Block Design test. The test measures difficulties in copying designs, making constructions and matching or discriminating patterns or faces. ${ }^{32}$ The participants have to use the blocks to reproduce seven patterns as quickly as possible. Each pattern has a maximum score from 2-7 and the summated score can therefore range between 0 and $51 .^{33}$

\subsection{Depression}

\subsubsection{Patient Health Questionnaire}

The Patient Health Questionnaire (PHQ-9) is based directly on the Diagnostic and Statistical Manual Fourth Edition (DSM-IV). It grades how often patients have been bothered by problems related to depression during the last 2 weeks. ${ }^{34}$ A scale ranging from not at all (0), to nearly every day (3) is used. PHQ-9 can be sub-grouped into no need of treatment for depression (0-4), mild depression (5-9), moderate depression (10-14), moderate to severe depression (15-19), and severe depression (20-27). The PHQ-9 and has showed good diagnostic validity. ${ }^{34}$

\subsection{Co-morbidities}

The Charlson comorbidity index was used for classifying co-morbidity. Nineteen co-morbid conditions were divided into 4 groups with weighted scores as follows: the first group included myocardial infarction, congestive heart failure, peripheral vascular disease, dementia, cerebrovascular disease, chronic lung disease, connective tissue disease, ulcer and chronic liver disease (assigned weight 1 ). The second group included hemiplegia, moderate or severe kidney disease, diabetes, diabetes with complication, tumor, leukemia, lymphoma 
(assigned weight 2). The third group included moderate or severe liver disease (assigned weight 3), and the fourth group included malignant tumor, metastasis, AIDS (assigned weight 6). The index score is the total of the assigned weights based on 1 -year mortality. The index has demonstrated satisfactory measurement properties with acceptable test-retest reliability. ${ }^{35}$

\section{Statistics}

Descriptive statistics were used to describe the study population. Continuous data were described with median and quartiles. Categorical data were described as frequencies and percentages. Differences between the groups were tested with Mann-Whitney U test as data were not normally distributed. Categorical data were analyzed with Pearson's chi-square test.

As the assumption of normality was violated, multiple linear regression analyses with robust standard errors were conducted to investigate if SDB and insomnia were associated with cognitive functioning. ${ }^{36}$ The cognitive tests were included as dependent variables while AHI, lowest saturation, saturation time below $90 \%$, insomnia and daytime sleepiness were included as independent variables. To adjust for other variables related to cognitive functioning, sex (male), age, education (university degree), Body Mass Index (BMI), B-type natriuretic peptide (BNP), diabetes and smoking were included as covariates, since they have previously been found to affect cognition. A power analysis based on $\alpha=.05,1-\beta=0.9, \mathrm{f} 2=.35$ and 11 independent variables showed that a sample size of 72 participants should be sufficient for the regression models. According to variance inflation factor, depression had a linear relationship to insomnia (VIF=2.1) and was therefore removed from the regression model. 
No other variables indicated problems with multicollinearity $(\mathrm{VIF}<2)$. A p-value of $\leq .05$ was considered statistically significant. SPSS version 20.0 (SPSS Inc, Chicago, IL, USA) was used for statistical analyses.

\section{Results}

\subsection{Population}

A total of 137 patients (68\% male, median age 72 years, 58\% NYHA II) were included in the study. Table 1 shows sample characteristics for the participants. Patients with SDB were older $(\mathrm{p} \leq .005)$, had worse functional capacity (i.e., scored in a higher NYHA class) $(\mathrm{p} \leq .022)$ had more atrial fibrillation $(\mathrm{p} \leq .001)$, higher creatinine values $(\mathrm{p} \leq .001)$ and used more diuretics $(\mathrm{p} \leq$ .03) compared to patients without SDB. The Charlson co-morbidity index showed no significant differences between those with and without SDB, and the low index mean value showed low risk for 1-year mortality in both groups. Patients with missing ApneaLink data $(\mathrm{n}=30)$ were older $(\mathrm{p} \leq .031)$, had higher creatinine values $(\mathrm{p} \leq .016)$ and used less B-blockers $(\mathrm{p} \leq .024)$ compared to those with full ApneaLink data $(n=107)$. None of the patients had documented or treated OSA or used continuous airway pressure ventilation therapy before study inclusion. 
Table 1 Sample characteristics of Heart Failure population (HF)

\begin{tabular}{|c|c|c|c|c|c|c|}
\hline & $\begin{array}{l}\text { Without } \\
\text { ApneaLink } \\
\text { n=30 }\end{array}$ & $\begin{array}{l}\text { With } \\
\text { ApneaLink } \\
\text { n=107 }\end{array}$ & p-value & $\begin{array}{l}\text { With } \\
\text { ApneaLink } \\
\text { No SDB } \\
\text { n=60 } \\
\end{array}$ & $\begin{array}{l}\text { With } \\
\text { ApneaLink } \\
\text { SDB } \\
\mathrm{n}=47 \\
\end{array}$ & p-value \\
\hline \multicolumn{7}{|l|}{ Characteristics } \\
\hline Age $\mathrm{Md}(\mathrm{Q} 1-3)$ & $77(65-83)$ & $71(63-78)$ & $.031^{a}$ & $67(60-76)$ & 74 (69-79) & $.005^{\mathrm{a}}$ \\
\hline Male n (\%) & $22(73)$ & $71(67)$ & $.312^{\mathbf{b}}$ & 38 (63) & $33(70)$ & $.209^{b}$ \\
\hline NYHA class & & & $.099^{\mathbf{b}}$ & & & $.022^{b}$ \\
\hline II n (\%) & $13(43)$ & $67(63)$ & & $42(70)$ & $25(53)$ & \\
\hline III n (\%) & $16(53)$ & $32(30)$ & & $13(22)$ & $19(40)$ & \\
\hline IV n (\%) & $1(3)$ & $3(3)$ & & $1(2)$ & $2(4)$ & \\
\hline Atrial fibrillation n (\%) & $11(37)$ & $44(41)$ & $.420^{b}$ & $23(38)$ & $21(45)$ & $<.001^{b}$ \\
\hline Diabetes n (\%) & $4(13)$ & $15(14)$ & $.556^{\mathbf{b}}$ & $7(12)$ & $12(25)$ & $.244^{\mathbf{b}}$ \\
\hline Pacemaker n (\%) & $10(33)$ & $30(28)$ & $.323^{\mathbf{b}}$ & $40(66)$ & $20(42)$ & $.235^{\mathbf{b}}$ \\
\hline Arterial hypertension $\geq 140 / 90 \mathrm{mmHg} \mathrm{n}(\%)$ & $8(27)$ & $34(31)$ & $.666^{\mathbf{b}}$ & $17(28)$ & $17(36)$ & $.148^{\mathrm{b}}$ \\
\hline Smoking n (\%) & $4(13)$ & $13(12)$ & $.220^{b}$ & $10(17)$ & $3(6.3)$ & $.122^{\mathbf{b}}$ \\
\hline Depression (PHQ9) mild to severe $\mathrm{n}(\%)$ & $14(47)$ & $39(36)$ & $.221^{\mathbf{b}}$ & $25(42)$ & $14(30)$ & $.239^{\mathbf{b}}$ \\
\hline Co-morbidity index Charlson Md (Q1-3) & $1(1-2)$ & $1(1-2)$ & $.304^{\mathbf{a}}$ & $1(1-2)$ & $2(1-3)$ & $.463^{\mathrm{a}}$ \\
\hline $0 \mathrm{n}(\%)$ & - & - & & - & - & - \\
\hline $1-2$ n (\%) & $24(80)$ & $87(81)$ & & $53(88)$ & $35(74)$ & \\
\hline 3-4 n (\%) & $6(20)$ & $16(15)$ & & $5(8)$ & $11(23)$ & \\
\hline$\geq 5$ n (\%) & - & $3(3)$ & & $2(3)$ & $1(2)$ & \\
\hline BMI Md (Q1-3) & $27(24-32)$ & $28(25-31)$ & $.556^{\mathrm{a}}$ & $27(25-31)$ & $30(25-34)$ & $.062^{\mathrm{a}}$ \\
\hline BNP Md (Q1-3) & $161(81-266)$ & $130(56-251)$ & $.483^{\mathrm{a}}$ & $119(44-231)$ & $160(79-275)$ & $.141^{\mathrm{a}}$ \\
\hline Creatinine Md (Q1-3) & $122(95-162)$ & $100(81-124)$ & $.016^{\mathrm{a}}$ & $88(77-109)$ & $111(88-143)$ & $.001^{\mathrm{a}}$ \\
\hline Medication & $\mathrm{n}=29$ & $\mathrm{n}=107$ & & $\mathrm{n}=59$ & $\mathrm{n}=45$ & \\
\hline ACEI n (\%) & $15(50)$ & $63(60)$ & $.241^{\mathbf{b}}$ & $37(62)$ & $26(57)$ & $.687^{\mathbf{b}}$ \\
\hline ARB n (\%) & $12(40)$ & $43(40)$ & $.573^{\mathbf{b}}$ & $24(41)$ & $20(44)$ & $.841^{\mathbf{b}}$ \\
\hline Aldosterone inhibitors n (\%) & $11(37)$ & $35(33)$ & $.426^{\mathbf{b}}$ & $20(33)$ & $15(33)$ & $1.00^{\mathbf{b}}$ \\
\hline B-blockers n (\%) & $24(80)$ & $99(93)$ & $.024^{b}$ & $55(93)$ & $45(100)$ & $.099^{\mathbf{b}}$ \\
\hline Diuretics n (\%) & $22(73)$ & $81(76)$ & $.463^{\mathrm{b}}$ & 41 (69) & $40(88)$ & $.030^{b}$ \\
\hline Digitalis n (\%) & $4(13)$ & $9(8)$ & $.310^{\mathbf{b}}$ & $6(10)$ & $3(7)$ & $.397^{\mathbf{b}}$ \\
\hline
\end{tabular}

\footnotetext{
${ }^{\mathbf{a}}$ Mann Whitney U test, ${ }^{\mathbf{b}}$ Chi-square- test
}

Note: No SDB $(A H I<15)$ and $\operatorname{SDB}(A H I \geq 15)$. NYHA= New York Heart Association, BMI= Body Mass Index, BNP= B-type natriuretic peptide, $\mathrm{ACEI}=$ Angiotensin converting inhibitor, $\mathrm{ARB}=$ Angiotensin II receptor blockers. 


\subsection{Sleep disordered breathing, sleep and wake patterns, insomnia and daytime sleepiness}

Respiratory variables, insomnia and daytime sleepiness are presented in Table 2. Forty-four percent of the 107 patients who underwent respiratory recordings had SDB. The SDB group had significantly deeper desaturations (i.e., a lower mean value for lowest saturation; $\mathrm{p}<.001$, more time below 90\%; $\mathrm{p}<.01)$, and more difficulties maintaining sleep $(\mathrm{p}<.05)$, compared to

patients without SDB. Neither MISS md score (4 vs. 5), lying down (8.47 hours vs. 8.36 hours), nor sleep duration (6.55 hours vs. 6.28 hours) differed between those with and without SDB. Occurrence of excessive daytime sleepiness was significantly more common among patients without SDB ( $\mathrm{p}<.001)$. Patients with missing ApneaLink data $(\mathrm{n}=30)$ had more excessive daytime sleepiness $(50 \%)(\mathrm{p}<.05)$. 
Table 2

Objective and subjective measures of breathing events, sleep and wake pattern, insomnia and excessive daytime sleepiness in chronic heart failure patients with and without sleep disordered breathing (SDB).

\begin{tabular}{|c|c|c|c|c|c|c|}
\hline & $\begin{array}{l}\text { Without } \\
\text { ApneaLink } \\
\text { n=30 }\end{array}$ & $\begin{array}{l}\text { With } \\
\text { ApneaLink } \\
\text { n=107 }\end{array}$ & p-value & $\begin{array}{l}\text { With } \\
\text { ApneaLink } \\
\text { No SDB } \\
\text { n=60 }\end{array}$ & $\begin{array}{l}\text { With } \\
\text { ApneaLink } \\
\text { SDB } \\
\mathbf{n}=47\end{array}$ & p-value \\
\hline $\begin{array}{l}\text { AHI } \\
\text { Md (Q1-3) }\end{array}$ & - & $13(5-25)$ & - & $7(3-11)$ & $26(21-37)$ & $<. \mathrm{O01}^{\mathrm{a}}$ \\
\hline $\begin{array}{l}\text { Lowest saturation } \\
\text { Md (Q1-3) }\end{array}$ & - & $85(78-88)$ & - & 87(83-89) & 80(74-85) & $<. \mathrm{O01}^{\mathrm{a}}$ \\
\hline $\begin{array}{l}\text { Time below saturation } \\
<90 \%\end{array}$ & - & $4(1-15)$ & - & $1(1-7)$ & $9(3-27)$ & $.003^{\mathrm{a}}$ \\
\hline $\begin{array}{l}\text { Minutes Md (Q1-3) } \\
\text { MISS Md (Q1-3) }\end{array}$ & $6(2-8)$ & $4(2-7)$ & $.145^{\mathrm{a}}$ & $4(2-6)$ & $5(3-8)$ & $.199^{\mathrm{a}}$ \\
\hline MISS 4 categories & & & $.440^{\mathrm{b}}$ & & & $.486^{\mathrm{b}}$ \\
\hline No insomnia n (\%) & $12(40)$ & $35(33)$ & & $24(40)$ & $14(30)$ & \\
\hline $\begin{array}{l}\text { Subclinical insomnia } \\
\mathrm{n}(\%)\end{array}$ & $6(20)$ & $38(35)$ & & $20(33)$ & $18(38)$ & \\
\hline $\begin{array}{l}\text { Moderate insomnia n } \\
\text { (\%) }\end{array}$ & $9(30)$ & $26(24)$ & & $12(20)$ & $14(30)$ & \\
\hline $\begin{array}{l}\text { Severe insomnia n } \\
\text { (\%) }\end{array}$ & $3(10)$ & $6(6)$ & & $5(8)$ & $1(2)$ & \\
\hline DIS n (\%) & $14(46)$ & $39(36)$ & $.221^{\mathrm{b}}$ & 23(38) & $16(34)$ & $.710^{\mathrm{b}}$ \\
\hline DMS n (\%) & 19(63) & $72(67)$ & $.396^{b}$ & $36(60)$ & $36(76)$ & $.046^{b}$ \\
\hline NRS n (\%) & 20(66) & $56(52)$ & $.127^{\mathrm{b}}$ & $28(47)$ & $28(30)$ & $.149^{\mathrm{b}}$ \\
\hline ESS totalscore Md (Q1-3) & $9.5(7-11)$ & $9(6-11)$ & $.656^{\mathrm{a}}$ & $9(6-12)$ & $8(5-12)$ & $.590^{\mathrm{a}}$ \\
\hline EDS n (\%) & $15(50)$ & $45(42)$ & $.050^{b}$ & $27(45)$ & $18(38)$ & $.001^{b}$ \\
\hline
\end{tabular}

${ }^{\mathrm{a}}$ Mann Withney U test, ${ }^{\mathrm{b}}$ Chi-square test.

Note: No SDB $(\mathrm{AHI}<15)$ and SDB(AHI $\geq 15)$. AHI-Apnoea-Hypopnoea Index, MISS -The Minimal Insomnia Symptom Scale, DIS-

Difficulties Initiating Sleep, 'DMS- Difficulties Maintaining Sleep, NRS- Non Restorative Sleep, EDS-Excessive Daytime Sleepiness,

ESS-The Epworth Sleepiness Scale 


\subsection{Cognitive function}

According to the MMSE seventy-eight percent of the whole study population had normal global cognitive function, $18 \%$ had mild cognitive dysfunction and $4 \%$ suffered risk of dementia (Table 3).

Measures of psychomotor speed, executive functions, visual-spatial perception/construct and memory, semantic and episodic memory and spatial abilities showed no differences between those with and without SDB. Patients with missing ApneaLink data $(n=30)$ had a significantly lower performance in semantic $(\mathrm{p}<.001)$ and spatial memory $(\mathrm{p}<.05)$. 
Table 3

Different areas of cognitive function in patients with chronic heart failure with and without sleep disordered breathing (SDB)

\begin{tabular}{|c|c|c|c|c|c|c|}
\hline & $\begin{array}{l}\text { Without } \\
\text { ApneaLink } \\
\mathrm{n}=30\end{array}$ & $\begin{array}{l}\text { With } \\
\text { ApneaLink } \\
\text { n=107 }\end{array}$ & p-value & $\begin{array}{l}\text { With } \\
\text { ApneaLink } \\
\text { No SDB } \\
\text { n=60 } \\
\end{array}$ & $\begin{array}{l}\text { With } \\
\text { ApneaLink } \\
\text { SDB } \\
\text { n=47 } \\
\end{array}$ & p-value \\
\hline $\begin{array}{l}\text { Global cognitive dysfunction }{ }^{\mathrm{C}} \\
\text { Md (Q1-3) }\end{array}$ & $28(27-30)$ & $29(27-29)$ & $.393^{\mathrm{a}}$ & $29(28-30)$ & $28(27-29)$ & $.446^{\mathrm{a}}$ \\
\hline $\begin{array}{l}\text { risk for dementia } \\
\mathrm{n}(\%)\end{array}$ & $1(3)$ & $4(4)$ & $.599^{\mathrm{b}}$ & $2(3)$ & $2(4)$ & $.261^{\mathrm{b}}$ \\
\hline $\begin{array}{l}\text { mild cognitive dysfunction } \\
\mathrm{n}(\%)\end{array}$ & $10(34)$ & $19(18)$ & $.323^{b}$ & $9(15)$ & $10(22)$ & $.526^{\mathrm{b}}$ \\
\hline $\begin{array}{l}\text { no cognitive dysfunction } \\
\text { n (\%) }\end{array}$ & $18(62)$ & $84(78)$ & $.357^{\mathrm{b}}$ & $49(82)$ & $35(74)$ & $.038^{b}$ \\
\hline $\begin{array}{l}\text { Psychomotorspeed }{ }^{\mathrm{d}} \\
\text { Md (Q1-3) }\end{array}$ & $38(24-51)$ & $38(27-57)$ & $.595^{\mathrm{a}}$ & $41(24-58)$ & $34(28-67)$ & $.952^{\mathrm{a}}$ \\
\hline $\begin{array}{l}\text { Executive function }{ }^{\mathrm{e}} \\
\text { Md (Q1-3) }\end{array}$ & $92(69-133)$ & $87(67-130)$ & $.433^{\mathrm{a}}$ & $86(67-124)$ & $89(68-137)$ & $.400^{\mathrm{a}}$ \\
\hline $\begin{array}{l}\text { Visual-spatial } \\
\text { Perception memory } \\
\text { Md (Q1-3) }\end{array}$ & $12(8-17)$ & $13(7-19)$ & $.277^{\mathrm{a}}$ & $15(9-20)$ & $11(4-17)$ & $.090^{\mathrm{a}}$ \\
\hline $\begin{array}{l}\text { Semantic memory }{ }^{\mathrm{g}} \\
\quad \text { Md (Q1-3) }\end{array}$ & $16(11-21)$ & $20(15-24)$ & $.001^{\mathrm{a}}$ & $22(18-26)$ & $20(14-24)$ & $.121^{\mathrm{a}}$ \\
\hline $\begin{array}{l}\text { Episodic memory }{ }^{\mathrm{h}} \\
\text { Md (Q1-3) }\end{array}$ & $3(2-6)$ & $4(3-6)$ & $.394^{\mathrm{a}}$ & $5(3-7)$ & $3(2-5)$ & $.153^{\mathrm{a}}$ \\
\hline $\begin{array}{l}\text { Spatial performance }{ }^{\mathrm{i}} \\
\text { Md (Q1-3) }\end{array}$ & $14(2-24)$ & $21(10-25)$ & $.033^{a}$ & $22(17-27)$ & $20(10-23)$ & $.140^{\mathrm{a}}$ \\
\hline
\end{tabular}




\subsection{Association between sleep and cognitive functioning}

The regression analysis showed that insomnia was significantly associated with decreased global cognitive dysfunction $(\mathrm{p}<.05)$ (Table 4). Although insomnia was significantly associated with cognitive functioning, this together with the rest of the included covariates explained a minor part of the total variance according to the $\mathrm{R}^{2}(.276)$. Higher age was associated with impaired executive functions $(\mathrm{p}<.05)$, visual-spatial perception/ construct memory $(\mathrm{p}<.01)$ and spatial performance $(\mathrm{p}<.001)$. Higher levels of educational degree showed a significant correlation with increased global cognitive function and semantic memory $(\mathrm{p}<.05)$. Smoking was associated with decreased semantic $(\mathrm{p}<.05)$ and episodic memory performance $(\mathrm{p}<.05)$. 
Table 4

Association between Sleep Disordered Breathing, insomnia and cognitive functioning (unstandardized regression coefficients).

\begin{tabular}{|c|c|c|c|c|c|c|c|}
\hline & $\begin{array}{l}\text { Global } \\
\text { cognitive } \\
\text { function, } \\
\text { screening for } \\
\text { dementia a }\end{array}$ & $\begin{array}{l}\text { Psychomotor } \\
\text { speed }^{\mathbf{b}}\end{array}$ & $\begin{array}{l}\text { Executive } \\
\text { function }^{c}\end{array}$ & $\begin{array}{l}\text { Visual-spatial } \\
\text { Perception } \\
\text { memory } \mathbf{d}\end{array}$ & $\begin{array}{l}\text { Semantic } \\
\text { memory }\end{array}$ & $\begin{array}{l}\text { Episodic } \\
\text { memory }\end{array}$ & $\begin{array}{l}\text { Spatial } \\
\text { performance }^{\mathbf{g}}\end{array}$ \\
\hline Model & $\mathrm{n}=\mathbf{1 0 0}$ & $\mathrm{n}=84$ & $\mathrm{n}=83$ & $n=83$ & $\mathrm{n}=86$ & $\mathrm{n}=86$ & $\mathrm{n}=86$ \\
\hline AHI & -.094 & -.147 & .177 & -.012 & .001 & -.025 & -.023 \\
\hline Time below $<90 \%$ & -.002 & .065 & .412 & -.018 & -.017 & -.009 & -.101 \\
\hline Insomnia(MISS) $^{\mathbf{h}}$ & $-.112 *$ & 1.391 & 2.710 & -.368 & -.036 & .012 & -.362 \\
\hline $\begin{array}{l}\text { Daytime } \\
\text { sleepiness(ESS) }\end{array}$ & .039 & -.729 & -1.216 & -.042 & .020 & .007 & .104 \\
\hline Male & .022 & 6.533 & 11.407 & 1.294 & -.694 & -.099 & -.680 \\
\hline Age & -.018 & .165 & $1.289 *$ & $-.265 * *$ & -.076 & -.051 & $-.414 * *$ \\
\hline $\begin{array}{l}\text { Education university } \\
\text { degree }\end{array}$ & $.875^{*}$ & .082 & -19.104 & 2.378 & $3.800 *$ & 1.302 & 3.499 \\
\hline BMI & .045 & -.719 & -1.443 & .165 & .140 & -.096 & .194 \\
\hline BNP & -.001 & .013 & -.031 & -.002 & -.002 & .000 & .003 \\
\hline Diabetes type I and II & -.678 & 3.209 & 8.190 & -.157 & .069 & -.801 & .233 \\
\hline Smoking & .099 & 2.872 & 3.501 & .395 & $1.793^{*}$ & $-.979 *$ & -1.166 \\
\hline Model R2 & .276 & .131 & .208 & .270 & .231 & .198 & .286 \\
\hline Model statistics & $\begin{array}{l}\mathrm{F}(11,88)=2.45 \\
\mathrm{p}=.010\end{array}$ & $\begin{array}{l}F(11,72)=1.21 \\
p=.296\end{array}$ & $\begin{array}{l}\mathrm{F}(11,71)=1.59 \\
\mathrm{p}=.121\end{array}$ & $\begin{array}{l}\mathrm{F}(11,71)=2.91 \\
\mathrm{p}=.003\end{array}$ & $\begin{array}{l}\mathrm{F}(11,74)=3.27 \\
\mathrm{p}=.001\end{array}$ & $\begin{array}{l}\mathrm{F}(11,74)=1.91 \\
\mathrm{p}=.051\end{array}$ & $\begin{array}{l}\mathrm{F}(11,74)=2.39 \\
\mathrm{p}=.013\end{array}$ \\
\hline
\end{tabular}

Measured with: ${ }^{\mathbf{a}}$ MMSE $=$ Mini Mental State Examination, ${ }^{\mathbf{b}}$ Trailmaking A, ${ }^{\mathbf{c}}$ Trailmaking B, ${ }^{\mathbf{d}}$ ROCF=Rey Ostereich Complex Figure, ${ }^{\mathbf{e}}$ Word Knowledge, ${ }^{\mathbf{f}}$ Memory of a story, ${ }^{\mathbf{g}}$ Block design,

${ }^{\mathbf{h}}$ MISS -The Minimal Insomnia Symptom Scale, ${ }^{\mathbf{i}}$ ESS-The Epworth Sleepiness Scale

Note: AHI= Apnoea-Hypopnoea Index, BMI= Body Mass Index, BNP= B-type natriuretic peptide. P-value $*<0.05, * *<0.01$ 


\section{Discussion}

This study examines the effects of SDB and sleep and wake pattern on cognitive impairment with an extensive neuropsychological test battery among elderly patients with CHF living in the community. The majority of the patients in our study population had mild to moderate CHF and almost half of them had SDB. An important finding was that the cognitive function seemed to be rather well preserved in these CHF patients, irrespective of whether SDB occurred or not. Surprisingly, we only found minor associations between SDB and cognitive function.

The SDB prevalence of $44 \%$ in our study is comparable to the $51 \%$ (i.e., $\mathrm{AHI} \geq 15$ in patients with $\mathrm{LVEF} \leq 40 \%$ ) and the $48 \%$ found by Oldenburg et al. ${ }^{1}$ and Damy et al., ${ }^{37}$ respectively. SDB-related breathing events have in previous studies been found to cause a disturbed sleep structure with increased arousals, ${ }^{38}$ insomnia, ${ }^{39}$ and daytime sleepiness. ${ }^{3}$ We found that patients with SDB had more difficulties maintaining sleep compared to those without SDB (76\% vs. 60\%, $\mathrm{p}<.05$ ), but sleep and wake pattern, as well as insomnia variables, did not differ between the groups. Excessive daytime sleepiness was even more common among those without SDB (45\% vs. 38\%, $\mathrm{p}<.01)$ and in the older group with missing ApneaLink data $(n=30)$. One explanation for this slightly surprising finding might be that SDB does not always present daytime symptoms. ${ }^{38}$ Furthermore, insomnia in patients with CHF might be caused by other disturbances than SDB, such as anxiety, dyspnea, dysrhythmias, coughing, deteriorations and side-effects. ${ }^{11,40}$ The sensitivity of the ESS might be low since CHF-related fatigue can be interpreted as excessive daytime sleepiness. An objective method to evaluate daytime sleepiness (i.e., Multiple Sleep Latency Test) could therefore be a more valid measure. $^{41}$ 
Mild and severe global cognitive impairment, as measured with the MMSE, was found in $18 \%$ and $4 \%$ of the total sample, respectively, but no differences were identified between those with and without SDB. Neither did measurements of psychomotor speed, executive functions, visual-spatial perception/construct and memory, semantic and episodic memory and spatial abilities. A recent study investigating sleep apnoea (i.e., diagnoses derived from medical records) and cognitive function in elderly heart failure patients found that co-morbid sleep may constitute a great risk for cognitive impairment (i.e., attention and executive functions). ${ }^{8}$ Other studies have found some type of cognitive impairment to occur among 25$80 \%$ of patients with $\mathrm{CHF}^{9,13}$ and SDB can be important with regards to attention, memory, executive functions and psychomotor speed. ${ }^{13,14}$ The mechanism for this is based on the hypothesis that apnoeic events ${ }^{13}$ and hypoxia ${ }^{12,14}$ following breathing events (i.e. apnoeas), are associated with sympathetic activation causing increased blood pressure, especially in patients with obstructive sleep apnoea. ${ }^{42}$ This might be of importance to the development of vascular dementia over time. We did not, despite the relatively high prevalence of SDB, find any significant associations between any SDB parameters and cognitive impairment. However, patients with missing ApneaLink data $(n=30)$ were older, had worse semantic memory and lower spatial performance compared to those with full ApneaLink data. Inversely, their cognitive deficits could be an explanation for the missing data. When examining an elderly population (i.e., mean age 65 years) with different co-morbidities (i.e., arthritis, high blood pressure, diabetes, renal disease, asthma and liver disease), Cohen-Zion ${ }^{43}$ found that only those with daytime sleepiness suffered from cognitive impairment. One explanation for the lack of association in the present study might be related to a potential referral bias. We included community- dwelling CHF patients who had been discharged from outpatient clinics. The Charlson co-morbidity index indicated that our sample had relatively few co-morbidities and, furthermore, there were no differences between those with and 
without SDB. Another explanation might be the relatively low number of patients suffering from deep desaturations and time below saturation $<90 \%$. Daytime sleepiness was also more common among those without SDB.

Insomnia may also affect memory and the ability to concentrate. ${ }^{44,45}$ The regression analyses showed that insomnia was associated with decreased global cognition. Higher age was associated with decreased executive function, visual-spatial perception and spatial performance. Higher education levels were important for improving global cognitive function and semantic memory, whereas smoking was related to impaired semantic and episodic memory. This is in line with previous findings in studies focusing on the elderly with and without cardiovascular disease. ${ }^{10,9,13}$ Depression is a factor commonly discussed to have an impact on both normal sleep and cognitive function. A recent study ${ }^{46}$ on community-dwelling older adults found, when using structural equation modeling, that cardiopulmonary symptoms (i.e., dyspnea, palpitations) and pain were directly associated with sleep disturbances, which led to an indirect association to depressive symptoms. Another recent study ${ }^{47}$ found that impaired cardiac function was associated with both inflammation and a symptom cluster describing sickness behavior (i.e., anhedonia, fatigue, and sleepiness). However, none of these studies measured cognitive function. In the present study depression was removed from the regression model since it had a linear relationship to insomnia, but in clinical situations it should be considered as a factor of importance for cognitive dysfunction.

It is possible to elaborate on our findings that cognitive function is well-preserved in elderly patients with mild to moderate CHF, without extensive co- or multi morbidity. There were no associations between SDB and cognitive function. These findings might decrease worries among health care personnel that SDB and cognitive problems are significant clinical problems among patients in NYHA class II with few co-morbidities. 
The design of the study was cross-sectional which limits the possibility to determine the causal effect between sleep disorders and cognitive dysfunction among the patients.

ApneaLink was used to collect data regarding SDB since it is a validated, ${ }^{18,19,44}$ feasible tool that is easy to use for elderly patients in home based settings. However, limitations include that it is not extensively used in CHF studies, it lacks the possibility to distinguish between obstructive and central sleep apnoea, as well as determine sleep onset and awakenings. A more advanced recording device (i.e., with six or more channels) could have provided more detailed information about these aspects, but since it would have been more complicated to use for an elderly patient it may have increased the level of missing data even more. On the other hand, an extensive test battery was used to measure cognitive function. This enabled us to measure different aspects of cognitive function but it might have caused an increased number of missing data. Despite a large proportion of missing ApneaLink data (i.e., due to patients having problems using the device and/or due to fatigue, and/or unwillingness to perform the recording), the sample size for all regression models was higher than the a priori estimated sample size of $n=72$. The sample size can therefore be judged as sufficient. Patients with missing data were more fatigued. Future studies should have a prospective, longitudinal design including elderly-elderly patients with more severe heart failure and more comorbidity.

\section{Conclusion}

The prevalence of cognitive dysfunction was low in this population with predominantly mild to moderate CHF of which $78 \%$ had a normal cognitive function. Almost half of the patients with CHF suffered from SDB. Difficulties maintaining sleep and excessive daytime sleepiness were associated with SDB. Insomnia was the only sleep-related factor significantly influencing cognition with more insomnia being related to poorer global cognitive function. 


\section{Acknowledgements}

Thomas Karlsson, CMIV, Linköping University for advice on cognitive instruments.

Funding by research grants from The Research Council in South-East Sweden (FORSS), Heart-Lung Foundation, Östergötland County Council, Linköping University and Linköping

University Hospital, Sweden.

\section{The authors declare no conflicts of interest}

\section{References}

1. Oldenburg O, Lamp B, Faber L, Teschler H, Horstkotte D, Topfer V. Sleep-disordered breathing in patients with symptomatic heart failure: a contemporary study of prevalence in and characteristics of 700 patients. Eur J Heart Fail. Mar 2007;9(3):251-257.

2. Arzt M, Young T, Finn L, Skatrud JB, Ryan CM, Newton GE, Mak S, Parker JD, Floras JS, Bradley TD. Sleepiness and sleep in patients with both systolic heart failure and obstructive sleep apnea. Arch Intern Med. Sep 18 2006;166(16):1716-1722.

3. Brostrom A, Stromberg A, Dahlstrom U, Fridlund B. Sleep difficulties, daytime sleepiness, and health-related quality of life in patients with chronic heart failure. J Cardiovasc Nurs. Jul-Aug 2004;19(4):234-242.

4. Johansson P, Arestedt K, Alehagen U, Svanborg E, Dahlstrom U, Brostrom A. Sleep disordered breathing, insomnia, and health related quality of life - a comparison between age and gender matched elderly with heart failure or without cardiovascular disease. Eur J Cardiovasc Nurs. Jun 2010;9(2):108-117.

5. $\quad$ Kalia M. Neurobiology of sleep. Metabolism. Oct 2006;55(10 Suppl 2):S2-6.

6. Killgore WD, Balkin TJ, Wesensten NJ. Impaired decision making following $49 \mathrm{~h}$ of sleep deprivation. J Sleep Res. Mar 2006;15(1):7-13.

7. Durmer JS, Dinges DF. Neurocognitive consequences of sleep deprivation. Semin Neurol. Mar 2005;25(1):117-129. 
8. Knecht KM, Alosco ML, Spitznagel MB, Cohen R, Raz N, Sweet L, Colbert LH, Josephson R, Hughes J, Rosneck J, Gunstad J. Sleep apnea and cognitive function in heart failure.

Cardiovascular psychiatry and neurology. 2012;2012:402079.

9. Pressler SJ. Cognitive functioning and chronic heart failure: a review of the literature (2002July 2007). J Cardiovasc Nurs. May-Jun 2007;23(3):239-249.

10. Kociuba C, Szabo A, Gunstad J, Spitznagel MB, Potter V, Hughes J, Waechter D, Josephson R, Rosneck J. Sleep and cognition in older adults with cardiovascular disease. J Cardiovasc Nurs. Nov-Dec 2010;25(6):497-502.

11. Garcia S, Alosco ML, Spitznagel MB, Cohen R, Raz N, Sweet L, Colbert L, Josephson R, Hughes $J$, Rosneck J, Gunstad J. Poor sleep quality and reduced cognitive function in persons with heart failure. Int J Cardiol. Apr 19 2012;156(2):248-249.

12. Zimmerman ME, Aloia MS. Sleep-disordered breathing and cognition in older adults. Current neurology and neuroscience reports. Oct 2012;12(5):537-546.

13. Yaffe K, Laffan AM, Harrison SL, Redline S, Spira AP, Ensrud KE, Ancoli-Israel S, Stone KL. Sleep-disordered breathing, hypoxia, and risk of mild cognitive impairment and dementia in older women. JAMA. Aug 10 2011;306(6):613-619.

14. Hrubos-Strom H, Nordhus IH, Einvik G, Randby A, Omland T, Sundet K, Moum T, Dammen T. Obstructive sleep apnea, verbal memory, and executive function in a community-based highrisk population identified by the Berlin Questionnaire Akershus Sleep Apnea Project. Sleep Breath. Mar 2012;16(1):223-231.

15. Reyes-Zuniga M, Castorena-Maldonado A, Carrillo-Alduenda JL, Perez-Padilla R, MartinezEstrada A, Gomez-Torres L, Torre-Bouscoulet L. Anxiety and depression symptoms in patients with sleep-disordered breathing. The open respiratory medicine journal. 2012;6:97-103.

16. Somers VK, White DP, Amin R, Abraham WT, Costa F, Culebras A, Daniels S, Floras JS, Hunt $\mathrm{CE}$, Olson LJ, Pickering TG, Russell R, Woo M, Young T. Sleep apnea and cardiovascular disease: an American Heart Association/American College Of Cardiology Foundation Scientific Statement from the American Heart Association Council for High Blood Pressure Research Professional Education Committee, Council on Clinical Cardiology, Stroke Council, and Council On Cardiovascular Nursing. In collaboration with the National Heart, Lung, and Blood Institute National Center on Sleep Disorders Research (National Institutes of Health). Circulation. Sep 2 2008;118(10):1080-1111.

17. McMurray JJ, Adamopoulos S, Anker SD, Auricchio A, Bohm M, Dickstein K, Falk V, Filippatos G, Fonseca C, Gomez.Sanchez MA, Jaarsma T, Kober L, Lip GY, Maggioni AP, Parkhomenko A, Pieske BM, Popescu BA, Ronnevik PK, Rutten FH, Schwitter J, Seferovic P, Stepinska J, Trindade PT, Voors AA, Zannad F, Zeiher A, E.S.C Committee for Practice Guidelines, Bax JJ, Baumgartner H, Ceconi C, Dean V, Deaton C, Fagard R, Funck-Bretano C, Hasdai D, Hoes A, Kirchhof P, Knuuti J, Kolh P, McDonagh T, Moulin C, Popescu BA, Reiner Z, Sechtem U, Sirnes PA, Tendera M, Torbicki A, Vahanian A, Windecker S, Document Reviewers, McDonagh $T$, Sechtem U, Bonet LA, Avraamides P, Ben Lamin HA, Brignole M, Coca A,Cowburn P, Dargie H, Elliot P, Flachskampf FA, Guida GF, Hardman S, Lung B, Merkely B, Mueller C, Nanas JN, Nielsen OW, Orn S, Parissis JT, Ponikowski P. ESC Guidelines for the diagnosis and treatment of acute and chronic heart failure 2012: The Task Force for the Diagnosis and Treatment of Acute and Chronic Heart Failure 2012 of the European Society of Cardiology. Developed in collaboration with the Heart Failure Association (HFA) of the ESC. Eur J Heart Fail. Aug 2012;14(8):803-869.

18. Erman MK, Stewart D, Einhorn D, Gordon N, Casal E. Validation of the ApneaLink for the screening of sleep apnea: a novel and simple single-channel recording device. J Clin Sleep Med. Jun 15 2007;3(4):387-392.

19. Nigro CA, Dibur E, Malnis S, Grandval S, Nogueira F. Validation of ApneaLink Ox for the diagnosis of obstructive sleep apnea. Sleep Breath. Mar 252012. 
20. Ancoli-Israel S, Cole R, Alessi C, Chambers M, Moorcroft W, Pollak CP. The role of actigraphy in the study of sleep and circadian rhythms. Sleep. May 1 2003;26(3):342-392.

21. Johns MW. A new method for measuring daytime sleepiness: the Epworth sleepiness scale. Sleep. Dec 1991;14(6):540-545.

22. Ulander M, Arestedt K, Svanborg E, Johansson P, Brostrom A. The fairness of the Epworth Sleepiness Scale: two approaches to differential item functioning. Sleep Breath. Feb 26 2012:Epub, ahead of print.

23. Broman JE, Smedje H, Mallon L, Hetta J. The Minimal Insomnia Symptom Scale (MISS): a brief measure of sleeping difficulties. Ups J Med Sci. 2008;113(2):131-142.

24. Drake CL, Roehrs T, Roth T. Insomnia causes, consequences, and therapeutics: an overview. Depress Anxiety. 2003;18(4):163-176.

25. Hellstrom A, Hagell P, Fagerstrom C, Willman A. Measurement properties of the Minimal Insomnia Symptom Scale (MISS) in an elderly population in Sweden. BMC geriatrics.

2010;10:84.

26. Crum RM, Anthony JC, Bassett SS, Folstein MF. Population-based norms for the Mini-Mental State Examination by age and educational level. JAMA. May 12 1993;269(18):2386-2391.

27. Hom J, Reitan RM. Neuropsychological correlates of rapidly vs. slowly growing intrinsic cerebral neoplasms. J Clin Neuropsychol. Aug 1984;6(3):309-324.

28. Reitan RM. Trail making Test. Tucson, AZ: Reitan Neuropsychological Laboratory; 1992.

29. Osterrieth P. Le test de copie dùne figure complexe. (trans J. Corwin and F.W. Bylsma (1993), The Clinical Neuropshychologist, 7, 9-15). 1944;30:206-356.

30. Dureman I, Kebbon,L, Österberg, E. Manual of the DS-Battery.Psykologi Förlaget, Stockholm; 1971.

31. Babcock RL, Salthouse TA. Effects of increased processing demands on age differences in working memory. Psychol Aging. Sep 1990;5(3):421-428.

32. Dureman I, Sälde H. Psykometrisk och Experimental-Psykologiska metoder för klinisk Tillämpning (Psychometric and Experimental-Psychological methods for Clinical Application). Uppsala, Sweden: Almqvist \& Wiksell; 1959.

33. Benton AL, Hécaen $\mathrm{H}$. Stereoscopic vision in patients with unilateral cerebral disease. Neurology. 1970;20:1084-1088.

34. Kroenke K, Spitzer RL, Williams JB. The PHQ-15: validity of a new measure for evaluating the severity of somatic symptoms. Psychosom Med. Mar-Apr 2002;64(2):258-266.

35. Charlson ME, Pompei P, Ales KL, MacKenzie CR. A new method of classifying prognostic comorbidity in longitudinal studies: development and validation. Journal of chronic diseases. 1987;40(5):373-383.

36. Juul S. An introduction to STATA for Health Research. 3 ed. Texas: College station, STATA press; 2010.

37. Damy T, Margarit L, Noroc A, Bodez D, Guendouz S, Boyer L, Drouot X, Lamine A, Paulino A, Rappeneau S, Stocia MH, Dubois-Rande JL, Andnot S, Hittinger L, d'Ortho MP. Prognostic impact of sleep-disordered breathing and its treatment with nocturnal ventilation for chronic heart failure. Eur J Heart Fail. Sep 2012;14(9):1009-1019.

38. Johansson P, Alehagen U, Svanborg E, Dahlstrom U, Brostrom A. Sleep disordered breathing in an elderly community-living population: Relationship to cardiac function, insomnia symptoms and daytime sleepiness. Sleep Med. Oct 2009;10(9):1005-1011.

39. Hayes DJr, Anstead MI, Ho J, Phillips BA. Insomnia and chronic heart failure. Heart Fail Rev. Sep 2009;14(3):171-182.

40. Brostrom A, Stromberg A, Dahlstrom U, Fridlund B. Patients with congestive heart failure and their conceptions of their sleep situation. Journal of advanced nursing. May 2001;34(4):520529. 
41. Chervin RD, Aldrich MS. The Epworth Sleepiness Scale may not reflect objective measures of sleepiness or sleep apnea. Neurology. Jan 1 1999;52(1):125-131.

42. Ballard RD. Sleep and medical disorders. Prim Care. Jun 2005;32(2):511-533.

43. Cohen-Zion M, Stepnowsky C, Marler, Shochat T, Kripke DF, Ancoli-Israel S. Changes in cognitive function associated with sleep disordered breathing in older people. J Am Geriatr Soc. Dec 2001;49(12):1622-1627.

44. Cormican LJ, Williams A. Sleep disordered breathing and its treatment in congestive heart failure. Heart. Oct 2005;91(10):1265-1270.

45. Brostrom A, Johansson P. Sleep disturbances in patients with chronic heart failure and their holistic consequences-what different care actions can be implemented? Eur J Cardiovasc Nurs. Sep 2005;4(3):183-197.

46. Johansson P, Riegel B, Svensson E, Broström A, Alehagen U, Dahlström U, Jaarsma T. The contribution of heart failure to sleep disturbances and depressive symptoms in older adults. Journal of geriatric psychiatry and neurology. Sep 2012;25(3):179-187.

47. Johansson P, Riegel B, Svensson E, Broström A, Alehagen U, Dahlström U, Jaarsma T. Sickness Behavior in Community-Dwelling Elderly Associations With Impaired Cardiac Function and Inflammation. Biological research for nursing. Nov 152012 [E-pub ahead of print]. 